anbiances Environnement sensible, architecture et espace urbain Comptes-rendus | 2016

\title{
Jean-Paul Thibaud. 2015. En quête d'ambiances : éprouver la ville en passant
}

Genève : MétisPresses

\section{Ola Söderström}

\section{(2) OpenEdition}

\section{Journals}

Édition électronique

URL : http://journals.openedition.org/ambiances/689

DOI : 10.4000/ambiances.689

ISSN : 2266-839X

Éditeur :

Direction Générale des Patrimoines - DAPA - MCC, UMR 1563 - Ambiances Architectures Urbanités (AAU)

\section{Référence électronique}

Ola Söderström, « Jean-Paul Thibaud. 2015. En quête d'ambiances : éprouver la ville en passant »,

Ambiances [En ligne], Comptes-rendus, mis en ligne le 14 avril 2016, consulté le 23 septembre 2020

URL : http://journals.openedition.org/ambiances/689; DOI : https://doi.org/10.4000/ambiances.689

Ce document a été généré automatiquement le 23 septembre 2020.

\section{cc) (†) $\ominus$}

Ambiances is licensed under a Creative Commons Attribution-NonCommercial-NoDerivatives 4.0 International License. 


\title{
Jean-Paul Thibaud. 2015. En quête d'ambiances : éprouver la ville en
}

\section{passant}

\author{
Genève : MétisPresses
}

\section{Ola Söderström}

\section{RÉFÉRENCE}

Thibaud, Jean-Paul. 2015. En quête d'ambiances: éprouver la ville en passant. Genève :

MétisPresses. 328 pages.

1 Si nous considérons, pour le seul plaisir de la typologie simpliste, qu'il y a deux sortes de chercheurs, les explorateurs qui changent souvent de territoire d'investigation et les creuseurs de sillon, Jean-Paul Thibaud est certainement à ranger du côté des seconds. Il travaille en effet la question des ambiances depuis de nombreuses années, seul, mais surtout en équipe avec ses collègues du CRESSON. Le beau livre qu'il publie aux éditions genevoises MétisPresses témoigne de ce labour répété du champ des ambiances au moyen de différents instruments de pensée et d'analyse empirique.

2 Cet ouvrage constitue une somme, au double sens du terme. D'une part, il s'agit, à ma connaissance, de l'ouvrage le plus complet à disposition sur la question des ambiances. D'autre part, il s'agit aussi d'une addition de textes : un recueil raisonné et organisé. Il y a dans ces deux acceptions de la somme les grandes qualités de cet ouvrage et ses quelques défauts: le livre fourmille de réflexions, d'idées et de créativité et il est parfois un peu redondant dans ses arguments et ses procédés.

3 En quête d'ambiances est structuré en cinq parties : la première analyse les fondements de la notion d'ambiance, la deuxième la mise en mot d'une ambiance, la troisième la teneur corporelle d'une ambiance urbaine, la quatrième la transformation des ambiances et la dernière les enjeux sociaux et urbains qui traversent cette notion. L'ambiance pour Thibaud c'est la « basse continue du monde sensible, la toile de fond à 
partir de laquelle s'actualisent nos perceptions et nos sensations ", autrement dit : un toujours-là de l'expérience, son enveloppe climatique. Il s'agit pour Jean-Paul Thibaud d'une notion transversale et interdisciplinaire. Elle permet de construire des ponts entre des pôles souvent disjoints dans l'analyse urbaine : la matérialité et la pratique sociale, le pré-cognitif et le cognitif, le ressenti et le représenté, le pathique et la pratique, le sentiment de soi et le sentiment du monde. Pour construire ainsi la notion d'ambiance - la monter en conceptualité - Thibaud se nourrit au fil de ses écrits d'une vaste et éclectique littérature. Elle va de la psychopathologie existentielle à l'ethnométhodologie, en passant par des théoriciens de la perception comme James Gibson et Tim Ingold. Cependant, la ressource théorique la plus récurrente dans cet ouvrage c'est le pragmatisme, en particulier dans sa version philosophique étatsunienne. S'il mobilise des pragmatistes contemporains comme Schusterman, Thibaud se réfère en effet principalement à John Dewey. On trouvera en particulier dans la dernière partie de l'ouvrage, plusieurs textes discutant les propositions de Dewey et leur pertinence pour la conceptualisation et l'analyse des ambiances. Cette posture conduit l'auteur à envisager l'ambiance comme procédant d'abord du ressenti, d'une logique vague, parce que pré-cognitive et comme devant être analysée en situation: l'« unité de base de toute expérience " pour Dewey. La lecture de Dewey inspire une approche des ambiances qui tente d'éviter l'intellectualisme, de se situer sous (ou avant) l'interprétation pour saisir une expérience au plus près de la corporéité, des émotions et des sensations.

Cette posture ouvre à la fabrication de méthodes qui prennent leurs distances avec les outils canoniques des sciences sociales qualitatives et en particulier avec l'entretien. C'est là l'un des grands intérêts de cet ouvrage. Outre la subtilité de la construction conceptuelle, une des qualités principales de cet ouvrage réside dans le fait qu'il témoigne de la grande créativité de l'auteur et de ses collègues du CRESSON dans l'élaboration de méthodes éthologiques d'observation des pratiques. Certes, les entretiens semi-directifs sont utilisés, mais rarement seuls et avec une conscience aigüe de leurs limites pour saisir les ambiances. La troisième partie de l'ouvrage offre ainsi, en puisant dans des recherches de terrain qui se focalisent alternativement sur les lieux et sur les pratiques qui y prennent place, des éléments très riches pour une analyse sensible des espaces urbains. Les lecteurs du livre trouveront ici beaucoup de sources d'inspiration en ce qui concerne la mise en œuvre de différentes méthodes, la pertinence de leurs combinaisons et, last but not least, l'art subtil et difficile de distiller les résultats produits par ces dispositifs.

En lisant avec appétit cet ouvrage, j'ai noté trois commentaires critiques. Le premier concerne la prudence ou la réserve de l'auteur en matière d'approche critique nourrie par ce qu'on pourrait appeler une économie politique des ambiances. À quelques endroits, une position critique sur la commercialisation des ambiances affleure. Mais celle-ci reste très furtive et peu approfondie. Or, il n'y a pas de raison à mon sens d'entretenir un partage entre économie politique urbaine et analyse sensible. Au contraire, l'économie politique critique, très présente jusqu'à être envahissante dans les études urbaines anglophones, gagnerait à être embarquée dans des analyses sensibles de l'urbain. Cette économie politique tend en effet à être répétitive, schématique et à préjuger des pratiques et des émotions sans prendre véritablement au sérieux leur analyse. Croiser une analyse situationnelle des ambiances avec celle de la production de plus en plus massive d'expériences à consommer - ce que certains appellent le «capitalisme cognitif» - permettrait à mon sens de renforcer les enjeux 
politiques de cette analyse, de montrer comment la production stratégique d'ambiances à des fins de profit ou de propagande modèle ou non l'expérience du monde sensible urbain. D'ailleurs, il s'agirait là d'un retour à l'une des sources de l'analyse des ambiances mentionnées par l'auteur: le situationnisme, pour laquelle l'analyse des ambiances était d'abord une critique, certes "naïve et volontariste " comme le relève Thibaud, mais une critique tout de même.

6 Le second point concerne l'absence du technologique. L'expérience de la ville, telle qu'elle se présente dans l'ouvrage est avant tout celle d'un marcheur dépourvu de prothèses techniques. Thibaud relève le caractère minoritaire de la marche aujourd'hui. Mais il ne discute pas le caractère limitatif de ce choix et n'aborde pas plus largement le rôle des médiations techniques dans l'expérience des espaces urbains. Or, et pardonnez-moi cette évidence, l'une des transformations essentielles dans l'expérience de la ville ces dernières décennies, réside dans le rôle accru des technologies numériques qui accompagnent, façonnent et redirigent les parcours urbains. Comment les ambiances se construisent-elles dans ces situations médiatisées et/ou dans des situations de parcours motorisés sont des questions sur lesquelles les lecteurs espéreront sans doute une seconde enquête de Thibaud dans les années à venir.

7 Enfin, il y a deux types de redondances dans cet ouvrage. D'abord, certains arguments et certaines citations apparaissent à plusieurs reprises. C'est évidemment fatal dans le cas d'un recueil de textes, à moins d'effectuer un gros travail de réécriture. Par ailleurs, ce ne sera pas un problème pour les lecteurs qui ne liront que quelques chapitres et ne les liront pas forcément dans la continuité. C'en est cependant un petit pour celui qui lit l'ouvrage dans sa continuité et qui chercherait une progression linéaire de l'argument. Avec ce recueil, nous voyons plutôt un travail de réflexion à l'œuvre, procédant par spirales et additions de nouvelles strates de l'enquête, ce qui est intéressant, mais produit quelques répétitions. La seconde forme de redondance est celle de la typologie. L'amour de la typologie est une sorte de marque de fabrique de l'auteur et plus généralement du laboratoire auquel il appartient. Il s'agit d'un procédé de raisonnement très utile puisqu'il crée de l'ordre, en l'occurrence ici dans les formes de l'expérience urbaine. En cela, il éclaire, produit de la compréhension. Dans une lecture continue de l'ouvrage, le recours à la typologie paraît toutefois un peu trop systématique. La typologie apparaît aussi parfois comme une fin en soi. Et on retrouve ici le souhait exprimé précédemment dans cette recension, à savoir que la grande finesse des analyses d'ambiances que contient ce livre soit couplée de façon plus explicite avec des enjeux sociaux et politiques et avec des dynamiques de transformation des espaces urbains.

8 Ces quelques réserves n'enlèvent rien aux nombreux mérites de ce livre. L'apport d'une lecture peut se mesurer par les types de notes que l'on prend. Dans ce cas, j'ai souligné de très nombreux passages, pris quatre pages de notes, mis des croix à de très nombreuses références de textes que je me suis promis de lire et établi une liste conséquente de pistes à suivre pour donner une place importante aux dimensions sensibles du phénomène urbain dans la recherche et l'enseignement. Ceci témoigne de la richesse et de l'importance de cette somme de réflexion et d'expérimentations sur les ambiances urbaines. 


\section{AUTEURS}

\section{OLA SÖDERSTRÖM}

Ola Söderström est professeur de géographie sociale et culturelle à l'Université de Neuchâtel. Ses travaux récents portent sur deux questions. D'une part, celle des dimensions de la mondialisation urbaine : en particulier, l'émergence de nouvelles typologies urbaines (notamment : M.

Guggenheim et O. Söderström, éd., Re-Shaping Cities : How Global mobility transforms architecture and urban form, London, Routledge, 2010 ; O. Söderström et al., éd., Critical Mobilities, London, Routledge, 2013) et la mobilité des politiques urbaines (notamment : Cities in Relations, Oxford, Wiley-Blackwell, 2014). D'autre part, il travaille aussi depuis peu sur les relations entre milieu urbain et psychose en collaboration avec des psychiatres et des ethnométhodologues (différents articles soumis à publication). 